\title{
Artificial neural networks based estimation of optical parameters by diffuse reflectance imaging under in vitro conditions
}

\author{
Mahmut Ozan Gökkan* and Mehmet Engin \\ Department of Electrical \& Electronics Engineering \\ Faculty of Engineering \\ Ege University Bornova, Izmir 35100, Turkey \\ *ozangokkan@gmail.com
}

Received 12 October 2015

Accepted 31 December 2015

Published 4 March 2016

\begin{abstract}
Optical parameters (properties) of tissue-mimicking phantoms are determined through noninvasive optical imaging. Objective of this study is to decompose obtained diffuse reflectance into these optical properties such as absorption and scattering coefficients. To do so, transmission spectroscopy is firstly used to measure the coefficients via an experimental setup. Next, the optical properties of each characterized phantom are input for Monte Carlo (MC) simulations to get diffuse reflectance. Also, a surface image for each single phantom with its known optical properties is obliquely captured due to reflectance-based geometrical setup using CMOS camera that is positioned at $5^{\circ}$ angle to the phantoms. For the illumination of light, a laser light source at $633 \mathrm{~nm}$ wavelength is preferred, because optical properties of different components in a biological tissue on that wavelength are nonoverlapped. During in vitro measurements, we prepared 30 different mixture samples adding clinoleic intravenous lipid emulsion (CILE) and evans blue (EB) dye into a distilled water. Finally, all obtained diffuse reflectance values are used to estimate the optical coefficients by artificial neural networks (ANNs) in inverse modeling. For a biological tissue it is found that the simulated and measured values in our results are in good agreement.
\end{abstract}

Keywords: Optical properties; diffuse reflectance; spectroscopy; Monte Carlo simulation; artificial neural networks.

\section{Introduction}

Determination of optical properties of a biological tissue is quite important in medical diagnosis and treatment. To characterize tissue conditions for the purposes of diagnosis of diseases, such as (pre) cancerous circumstances, or for monitoring response to treatment, noninvasive diffuse reflectance imaging approach plays a key role. This is because optical

${ }^{*}$ Corresponding author.

This is an Open Access article published by World Scientific Publishing Company. It is distributed under the terms of the Creative Commons Attribution 4.0 (CC-BY) License. Further distribution of this work is permitted, provided the original work is properly cited. 
properties of a tissue may reveal information concerning the morphological and biochemical composition of that tissue. ${ }^{1}$

Therefore, the calculation of tissue optical parameters through noninvasive imaging should be reliable when repeatedly performing measurements. ${ }^{1}$

Tissues can be characterized by their optical properties, which are defined by absorption coefficient $\left(\mu_{a}\right)$, the scattering coefficient $\left(\mu_{s}\right)$, anisotropy factor $(g)$ and reduced scattering coefficient $\left[\mu_{s}^{\prime}=\mu_{s}(1-g)\right]$. $^{1,2}$ Since Boltzmann based radiative transfer equations (RTE) are nontrivial, diffusion approximation is more suitable than RTE equations. ${ }^{3}$ However, due to the fact that the solution of diffusion equation is still too complex to calculate diffuse reflectance depending on absorption and scattering coefficients, another way to obtain diffuse reflectance is Monte Carlo (MC) approach in forward modeling. Monte Carlo simulations are more efficient than the classical diffusion equation. Statistical modeling based MC simulations rely on the calculation of an expected value of all random samples for multi-photons in a biological tissue with iterative approach. ${ }^{4}$ In this study, both experimental and simulative approaches are considered for getting diffuse reflectance parameter depending on source-detector distance in millimeter.

In experiments, all measurements were taken into account in terms of the use of noninvasive diffuse optical two-dimensional (2D) imaging and of spectrometer for optical characterization of a turbid medium. For doing this, transmission spectroscopy was firstly used for an experimental setup to measure the coefficients. Then, in reflectance-based geometrical setup, CMOS camera was positioned at $5^{\circ}$ angle to the phantoms for oblique image capturing. Thereby, images were taken from the phantoms that were previously characterized via the transmission-based spectroscopic measurements. For the illumination of light, a laser source at $633 \mathrm{~nm}$ wavelength is preferred, because optical properties of tissue chromophores on that wavelength are nonoverlapped. ${ }^{5} \mathrm{With}$ a different approach, we can suggest that CMOS camera-based measurements can be an alternative way for getting optical parameters. Therefore, a measuring instrument can be miniaturized using $2 \mathrm{D}$ arrays. An algorithmic way on how to obtain diffuse reflectance (2D arrays) through images will be given in our materials and methods in Sec. 3.

In our study, artificial neural networks (ANNs) were also used to obtain optical parameters such that absorption and scattering coefficients were estimated using diffuse reflectance. ${ }^{2}$ Also MC simulations were performed for the sake of possibly obtaining simulated diffuse reflectance, to contribute to the ANN as input parameters. Moreover, captured images from prepared phantoms were considered for ANN structure. In terms of building a good prediction models for ANN, we elaborated on stabilizing the power of laser light and then capturing surface images that have high spatial resolution. The Canon EOS 40D CMOS camera we used during imaging to acquire high spatially resolved images has the $3888 \times 2592$ pixels and a metric distance between two pixels of $5.7 \mu \mathrm{m}$. In addition, in $\mathrm{MC}$ simulation that we coded for a single layered skin-tissue to model transporting multi-photons,${ }^{6}$ it involves no physical approximations but produces estimates of the reflectance through statistical modeling. At this point, our $\mathrm{C}$ programming language code named "distance_based_reflectance" was fine modeled in terms of practicality, reliability and suitability to perform MC simulations. Also, in terms of well-modeled ANN structure, optimum algorithm such as Levenberg-Marquardt and a number of hidden layers were selected.

\section{Theoretical Background}

As it is known that a tissue consists of four main layers that are, from top to deep; stratum corneum, epidermis, dermis and subcutaneous. Each layer has a thickness that can be changeable from person to person. ${ }^{7}$ The thickness of an each layer is generally fixed for the sake of simplicity in a simulative approach. As a real life scenario, most biological tissues are characterized by their optical properties such as absorption and scattering coefficients. ${ }^{8}$ Absorption coefficient mainly means that the probability of photon being absorbed per unit path length in a medium. Likewise, scattering coefficient defines that the probability of photon being scattered per unit path length in a medium. At this point, if a medium has strong scatters, that medium is referred to as scattering media or turbid media. ${ }^{1}$

\subsection{Forward modeling}

Photon migration or light propagation in biological tissue can be analytically modeled using RTE as given in Eq. (1) or numerically modeled with MC simulations. Because the RTE is difficult to solve, it 
is often approximated to a diffusion equation, which provides solutions that are more computationally efficient but less accurate than those provided by the MC method. ${ }^{1}$

$$
\begin{aligned}
\frac{\partial L(\mathbf{r}, \hat{s}, t)}{\partial t}= & -\hat{s} \cdot \nabla L(\mathbf{r}, \hat{s}, t)-\mu_{t} L(\mathbf{r}, \hat{s}, t) \\
& +\mu_{s} \int L(\mathbf{r}, \hat{s}, t) \cdot P\left(\hat{s}^{\prime} \cdot \hat{s}\right) d \Omega^{\prime} \\
& +S(\mathbf{r}, \hat{s}, t),
\end{aligned}
$$

where $L(\mathbf{r}, \hat{s}, t)$ is radiance, $\mathbf{r}$ : location, $\hat{s}$ : direction vector and $t$ : time. $\mu_{t}$ is total attenuation coefficient. The product $P\left(\hat{s}^{\prime} \cdot \hat{s}\right) d \Omega^{\prime}$ is scattering phase function and represents the probability of light with propagation direction $\hat{s}^{\prime}$ being scattered into $d \Omega^{\prime}$, which is a unit solid angle, around direction $\hat{s}$. Also, $S(\mathbf{r}, \hat{s}, t)$ is a light source. As it is seen in Eq. (1), it contains more independent and unknown variables and is analytically difficult to solve. Also the equation may not produce desired values as accurate as possible. ${ }^{9}$ Instead, in terms of modeling photon propagation in a medium, there are two effective ways to get easier and more accurate resultant parameters that can diffuse reflectance and transmittance. At this point, diffuse term is based on a banana model when light or a photon travels in a medium and back propagates to the top of same surface regarding source-detector distance. Thereby, in diffuse regime, MC simulation can be used to obtain an expected value of all random variables that are generated by computer for multi-photons with iterative approach. This expected value can be considered as diffuse reflectance that they depend on how source-detector pair is positioned. In MC modeling, we have preconditioned for the modeling of single layered tissue that was assumed to be a semi-infinite highly scattering medium. Although the main disadvantage of iterative MC technique is time consuming, graphics processing unit (GPU) can be used to reduce long execution time of the simulations. ${ }^{10,11}$ Yong et al. ${ }^{10}$ and Cserkaszky ${ }^{11}$ used GPU-based simulations to produce fast results in their studies.

\subsection{Inverse modeling}

Artificial neural networks based on statistical learning such as supervised and unsupervised learning. Basically, unsupervised learning provides how a network structure can learn to represent particular input patterns in a way that reflects the statistical structure of the overall collection of input patterns. On the other hand, supervised learning consists of presenting an input pattern and a desired pattern to the output layer and updating network parameters (e.g., weights) in order to produce actual output more similar to the desired one. ${ }^{18}$ For example, backpropagation is the most widely used algorithm for supervised learning with multi-layered feed forward networks ${ }^{23}$ and its basic approach is based on a chain rule to compute the effect of weights in the network with respect to an error (cost) function $E$;

$$
\frac{\partial E}{\partial w_{i j}}=\frac{\partial E}{\partial s_{i}} \frac{\partial s_{i}}{\partial n e t_{i}} \frac{\partial n e t_{i}}{\partial w_{i j}},
$$

where $w_{i j}$ is the weight from neuron $j$ to neuron $i, s_{i}$ is the output and net $_{i}$ is the weighted sum of the inputs of neuron $i$. The aim of using partial derivative for each weight in Eq. (2) is to minimize cost function that is achieved by performing a gradient descent;

$$
w_{i j}(t+1)=w_{i j}(t)-\alpha \frac{\partial E}{\partial w_{i j}}(t)
$$

where $\alpha$ is the learning rate, which scales the derivative and it has an important effect on time needed until convergence is reached for updating weights in Eq. (3). In our study, backpropagation algorithm was used to estimate the optical coefficients using diffuse reflectance values in a way of inverse modeling.

\section{Materials and Methods}

\subsection{Monte Carlo simulation}

Statistical modeling based MC approach relies on the calculation of an expected value of randomly generated samples (or numbers) between 0 and 1 . In the field of biomedical optics, many different MC simulations are performed for the migration of multi-photons in a biological tissue with iterative approach. ${ }^{4}$ Because of the fact that there are no international standards to model a complex skintissue and no exact analytical solution exists for the problem of light propagation in turbid media, ${ }^{5}$ we coded a $\mathrm{MC}$ simulation using $\mathrm{C}$ programming language in terms of practicality and reliability. All simulations were based on a flowchart that was taken from Ref. 7 as shown in Fig. 1. The main aim 


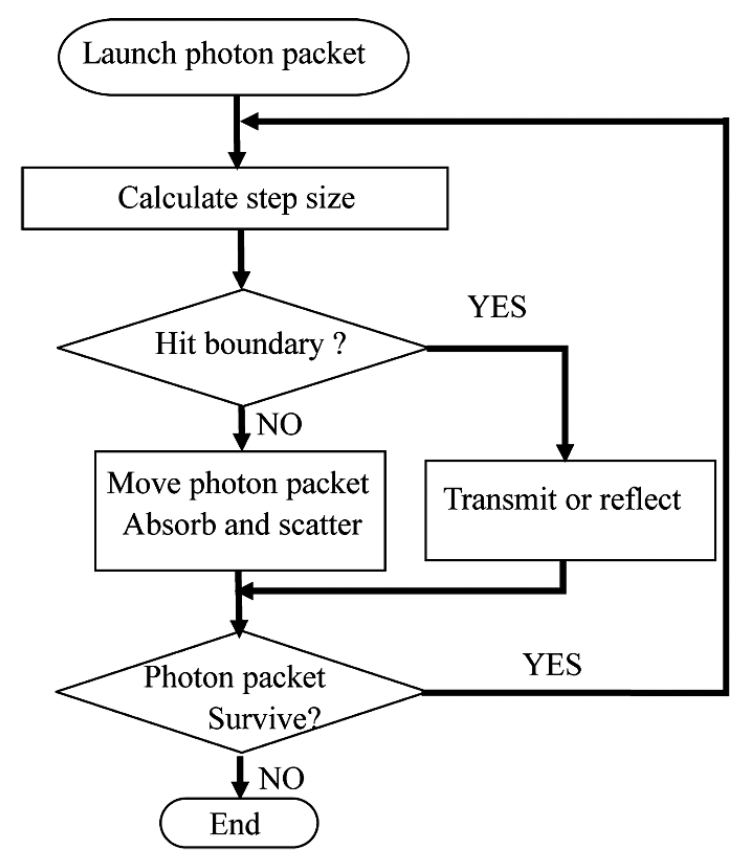

Fig. 1. A flowchart of the MC simulation.

of the simulation is to produce a diffuse reflectance identifying some boundary conditions and required inputs. We considered a semi-infinite scattering medium that is only defined by top and bottom surface. The left and right boundaries of a medium are not taken into account for a semi-infinite condition. Also, in our model, photons were positioned at the top of the medium and required input parameters were handled. The parameters are absorption coefficient $\left(\mu_{a}\right) \mathrm{mm}^{-1}$, scattering coefficient $\left(\mu_{s}\right) \mathrm{mm}^{-1}$, anisotropy factor $(g)$ and the ratio of refractive indices between medium and air was fixed at 1.4. Also, each single photon was initialized with its packet (or weight) which is 1 for the sake of a simple modeling of light-medium interaction.

Each simulation was executed for 10,000 photons and the Henyey-Greenstein scattering phase function was used in which the angular dependence is described by solely one parameter, $g .{ }^{13}$ Anisotropy factor $(g)$ or the mean cosine of scattering angle was fixed at 0.8 for all simulations. If a photon enters a medium under boundary conditions, absorption and mostly scattering events will be occurred due to highly scattering in that medium. In diffuse regime, we took the backscattered photons in Fresnel reflection approach due to banana model and then an expected value of all acquired photons with their remaining packets (or weights) was calculated.
Fresnel reflection for surface scattering effects of a medium can be calculated for smooth surfaces using Fresnel's equation that describes what fraction of light is reflected, ${ }^{24}$

$$
R_{f}=\frac{1}{2} x \frac{(a-c)^{2}}{(a+c)^{2}} x\left\{1+\frac{[c(a+c)-1]^{2}}{[c(a-c)+1]^{2}}\right\},
$$

where $R_{f}$ is the Fresnel reflection, $c=\cos \left(\theta_{i}\right), \theta_{i}$ is the angle of incident light, $a=n^{2}+c^{2}-1$ and $n$ is the refractive index of tissue.

\subsection{Experimental measurements}

We employed three main approaches that the first one was setting up an optical assembly for measurements, the latter was MC simulation for forward modeling and finally, we used an ANN structure to apply inversion procedure. In experimental setup, a 2D image of each single prepared sample was acquired using CMOS integrated canon EOS 40D camera that contains interchangeable digital single lens. Camera was fixed at $5^{\circ}$ angular position for oblique capturing. Also, laser light source at $633 \mathrm{~nm}$ wavelength was used to illuminate the samples and laser power was fixed at $420 \mathrm{~mW}$.

As it is seen in Fig. 2, we used a mirror that reflects laser light which falls perpendicularly onto a prepared sample to capture reflected photons through the camera. After we prepared 30 different mixture samples (liquid phantoms) that include $20 \% 500 \mathrm{ml}$ clinoleic intravenous lipid emulsion (CILE) and EB powder dye solvent. In addition, CILE type lipid emulsion contains 2.25 gram glycerol, 4 gram soybean oil, 1.2 gram egg phospholipids, 4 gram essential fatty acids and 16 gram olive oil per $100 \mathrm{ml}$. The optical properties for each sample are determined using transmission-based USB4000 UV-VIS Ocean Optics type spectrometer as shown in Fig. 3. Then we input the obtained optical properties $\left(\mu_{a}\right.$ and $\left.\mu_{s}\right)$ of the samples for MC simulations to produce diffuse reflectance. The general structure of this study can be seen in Fig. 4.

To prepare a mixture sample using EB dye and CILE type lipid, as shown in Table 1, for example, we added $100 \mathrm{ml}$ deionized water into $0.2 \mathrm{ml}$ CILE without adding EB dye for the first sample. Hence, we can easily determine the optical properties of that CILE using Eq. (5), because we know that water is a weak absorber in neglecting scattering factor and its absorption coefficient at $633 \mathrm{~nm}$ 


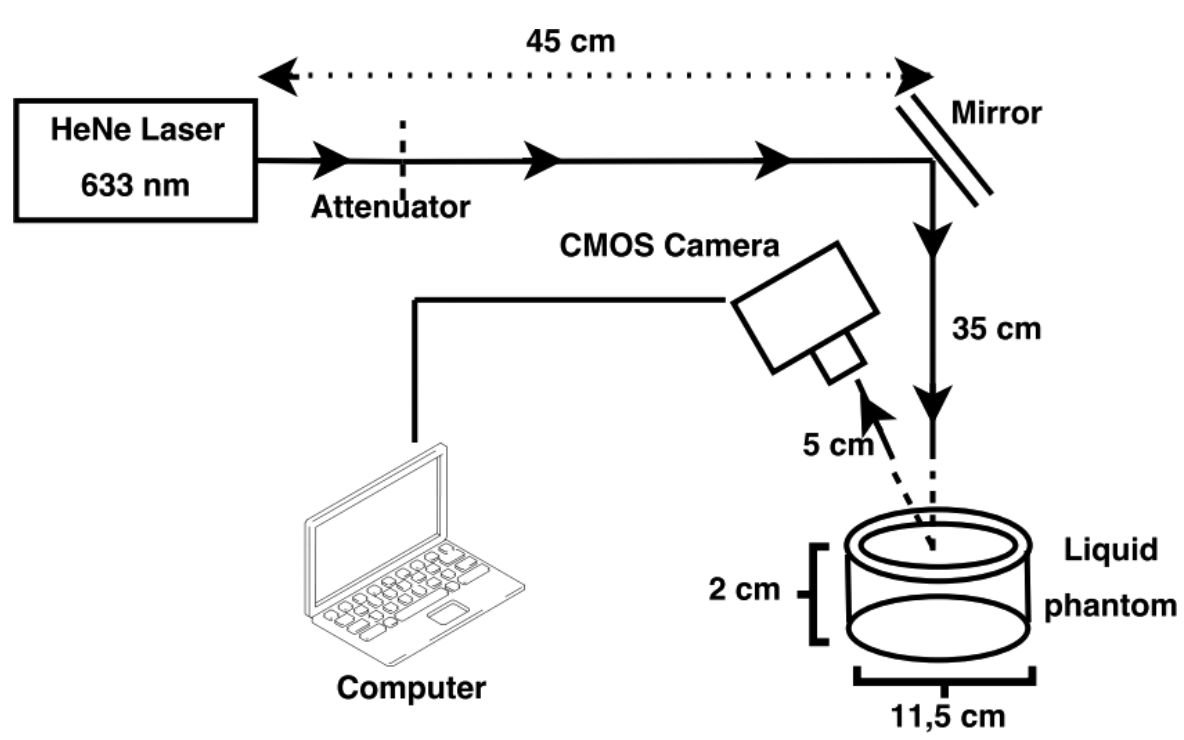

Fig. 2. Diffuse reflectance-based experimental setup for surface imaging of the phantoms.

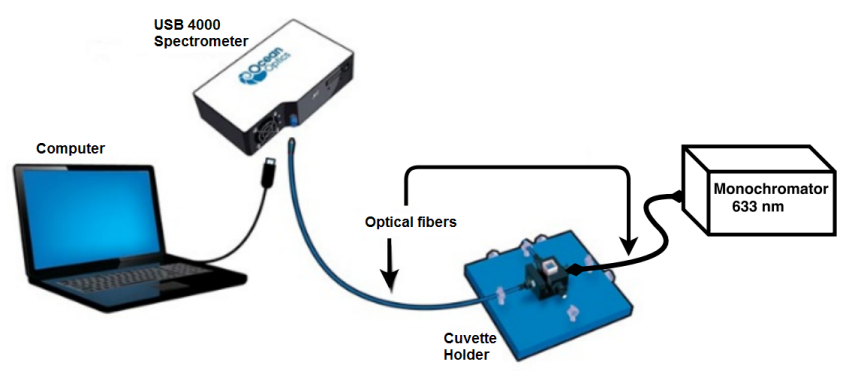

Fig. 3. Transmission-based experimental setup for spectroscopic measurements as the results shown in Table 1. (Cuvette thickness is $0.5 \mathrm{~cm})$.

wavelength is $0.00326 \mathrm{~cm}^{-1}$ from Ref. 19. Moreover, CILE is also a strong scatterer supposing that it contains no absorption property. ${ }^{16}$ Thereby, we assumed that the lipid is a scatterer and EB dye is an absorber. For the second sample, we added $1 \mathrm{mg}$ same dye into the first sample and then, depending upon the calculated total attenuation coefficient $\left(\mu_{t}\right)$ in Eq. (5), the absorption coefficient of the dye can be extracted. ${ }^{14}$ Thereby, optical properties of all phantoms were calculated in this way by adding method..$^{15}$

$$
\mu_{t}=\frac{1}{\log (e)} \frac{A_{T}}{d},
$$

where $d$ is cuvette thickness, $0.5 \mathrm{~cm}$. $A_{T}$ is transmission-based absorbance and $\mu_{t}$ is the total attenuation coefficient. After identifying the optical properties of all samples (liquid phantoms) using transmission-based spectrometer, the measured optical properties were then used as input parameters which are absorption coefficient and scattering coefficient for MC simulations that output diffuse reflectance. Next, the following steps for getting diffuse reflectance values from captured image have been respectively done in algorithmic way for 30 different samples image through liquid phantoms;

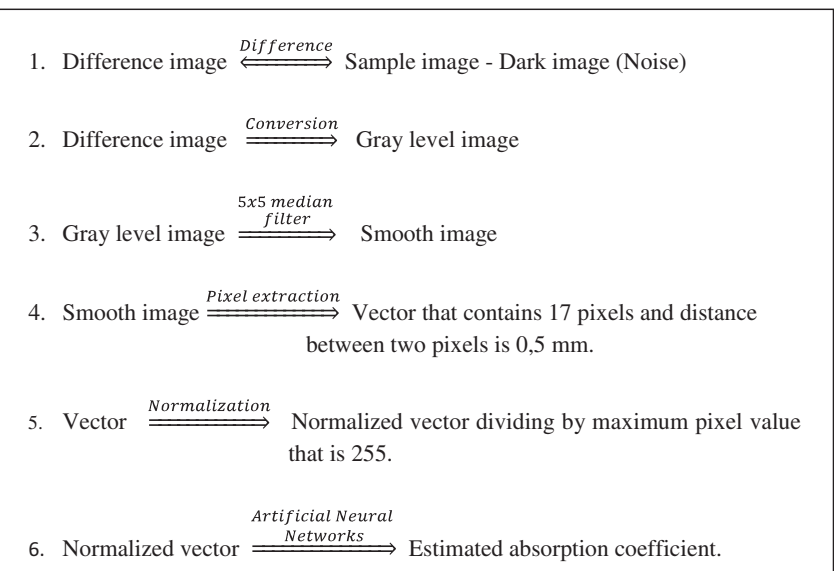

As seen in the algorithm mentioned above, ANN was used to estimate an optical absorption coefficient through corresponding imagebased normalized vector. The main aim of obtaining images is to estimate an optical property of a biological tissue imitated liquid phantom using $\mathrm{ANN}$ is to make experimental setup easily without the need of spectrometer. 


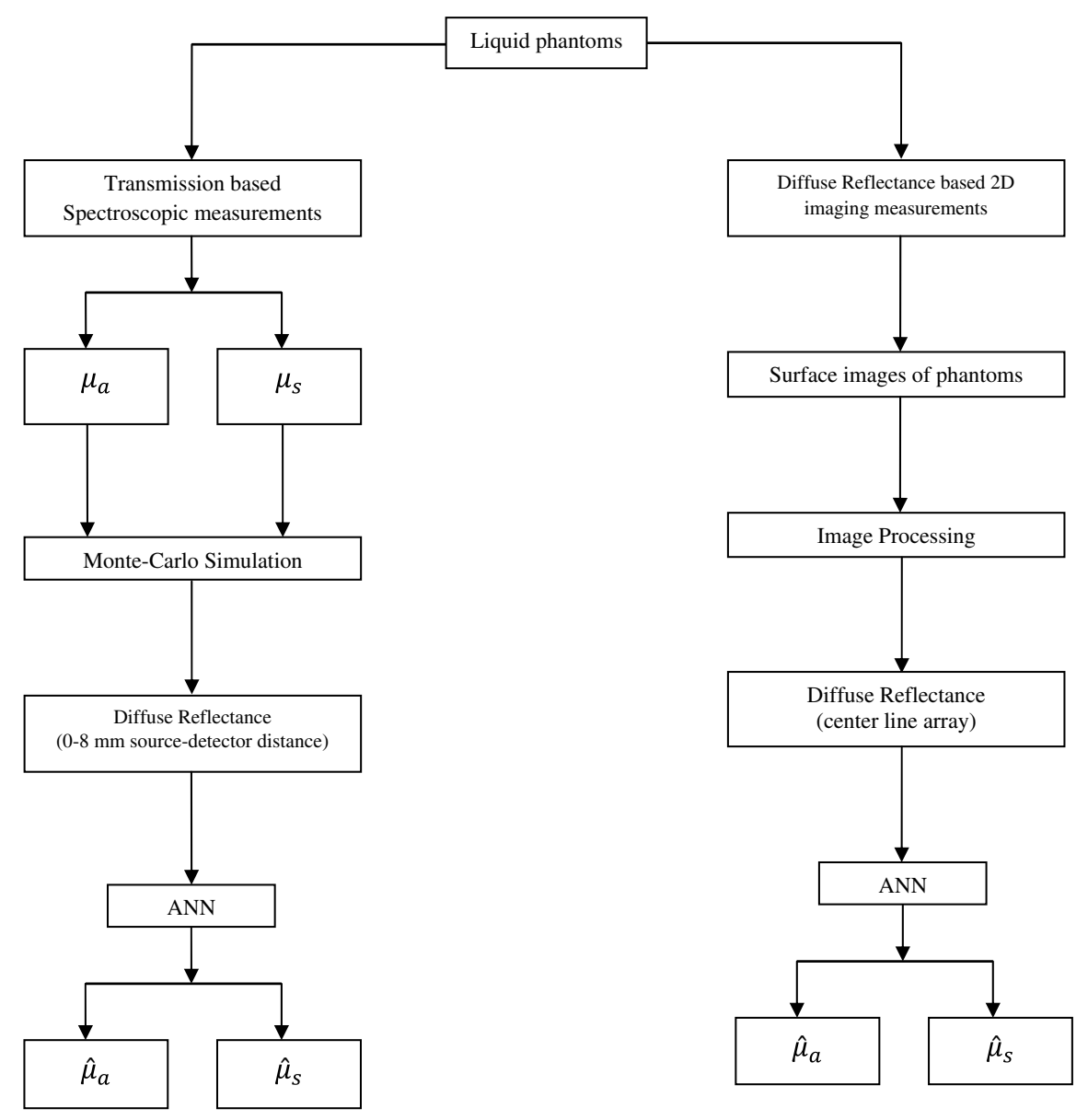

Fig. 4. General flowchart of this study.

Table 1. Liquid Phantoms (in vitro) prepared for getting optical characterizations.

\begin{tabular}{cccccccc}
\hline Phantoms & $\mathrm{V}_{\text {water }}(\mathrm{ml})$ & $V_{\text {lipid }}(\mathrm{ml})$ & Evans Blue $(\mathrm{mg})$ & Absorbance & $\mu_{t}(\mathrm{~cm})^{-1}$ & $\mu_{s}(\mathrm{~cm})^{-1}$ & $\mu_{a}(\mathrm{~cm})^{-1}$ \\
\hline 1 & 100 & 0.2 & 0 & 0.311 & 1.4322 & 1.4289 & 0.00326 \\
2 & 100 & 0.2 & 1 & 0.673 & 3.0992 & 1.4289 & 1.667 \\
3 & 100 & 0.2 & 2 & 1.123 & 5.1716 & 1.4289 & 3.7394 \\
4 & 100 & 0.2 & 3 & 1.534 & 7.0643 & 1.4289 & 5.6321 \\
5 & 100 & 0.2 & 4 & 1.884 & 8.6761 & 1.4289 & 7.2439 \\
6 & 100 & 0.2 & 5 & 2.230 & 10.2695 & 1.4289 & 8.8373 \\
7 & 100 & 0.2 & 10 & 3.115 & 14.3451 & 1.4289 & 12.9129 \\
8 & 100 & 0.2 & 15 & 3.144 & 14.4787 & 1.4289 & 13.0465 \\
9 & 100 & 0.4 & 0 & 0.834 & 3.8407 & 3.8374 & 0.00326 \\
10 & 100 & 0.4 & 1 & 1.346 & 6.1985 & 3.8374 & 2.3578 \\
11 & 100 & 0.4 & 2 & 1.916 & 8.8235 & 3.8374 & 4.9828 \\
12 & 100 & 0.4 & 3 & 2.323 & 10.6978 & 3.8374 & 6.8571 \\
13 & 100 & 0.4 & 4 & 2.777 & 12.7886 & 3.8374 & 8.9479 \\
14 & 100 & 0.4 & 5 & 2.862 & 13.1800 & 3.8374 & 9.3393 \\
15 & 100 & 0.6 & 0 & 1.172 & 5.3972 & 5.3939 & 0.00326 \\
16 & 100 & 0.6 & 1 & 1.756 & 8.0867 & 5.3939 & 2.6895 \\
17 & 100 & 0.6 & 2 & 2.058 & 9.4775 & 5.3939 & 4.0803 \\
18 & 100 & 0.6 & 3 & 2.331 & 10.7347 & 5.3939 & 5.3375 \\
19 & 100 & 0.6 & 4 & 2.705 & 12.4570 & 5.3939 & 7.0598 \\
\hline
\end{tabular}


Table 1. (Continued)

\begin{tabular}{cccccccc}
\hline Phantoms & $\mathrm{V}_{\text {water }}(\mathrm{ml})$ & $V_{\text {lipid }}(\mathrm{ml})$ & Evans Blue $(\mathrm{mg})$ & Absorbance & $\mu_{t}(\mathrm{~cm})^{-1}$ & $\mu_{s}(\mathrm{~cm})^{-1}$ & $\mu_{a}(\mathrm{~cm})^{-1}$ \\
\hline 20 & 100 & 0.6 & 5 & 2.861 & 13.1754 & 5.3939 & 7.7782 \\
21 & 100 & 0.6 & 10 & 2.965 & 13.6544 & 5.3939 & 8.2572 \\
22 & 100 & 0.6 & 15 & 2.967 & 13.6636 & 5.3939 & 8.2664 \\
23 & 100 & 1 & 0 & 1.553 & 7.1518 & 7.1485 & 0.00326 \\
24 & 100 & 1 & 1 & 1.772 & 8.1604 & 7.1485 & 1.0086 \\
25 & 100 & 1 & 2 & 2.519 & 11.6004 & 7.1485 & 4.4486 \\
26 & 100 & 1 & 3 & 2.785 & 12.8254 & 7.1485 & 5.6736 \\
27 & 100 & 1 & 4 & 2.896 & 13.3366 & 7.1485 & 6.1848 \\
28 & 100 & 1 & 5 & 2.940 & 13.5392 & 7.1485 & 6.3874 \\
29 & 100 & 1 & 10 & 2.963 & 13.6452 & 7.1485 & 6.4934 \\
30 & 100 & 1 & 15 & 2.963 & 13.6452 & 7.1485 & 6.4934 \\
\hline
\end{tabular}

\section{Results and Discussion}

In all measurements, we saw that the scattering coefficients gradually increased and likewise, absorption coefficients of dye increased while adding dye. For example, we fixed the concentration of lipid and added dye for the first eight phantoms as seen in Table 1. In the same manner, the other phantom groups, such as 9 th to 14 th, 15 th to 22 nd and $23 r d$ to 30 th, were made and each group has the fixed lipid concentration. Scattering coefficients of each group have also a fixed value due to the fixed concentration of lipid in each group and the absorption coefficients increased for each phantom, because lipid has a weak absorption property (because of strong absorption property for evans blue (EB) dye). On the other hand, MC simulations were performed for 30 liquid samples that

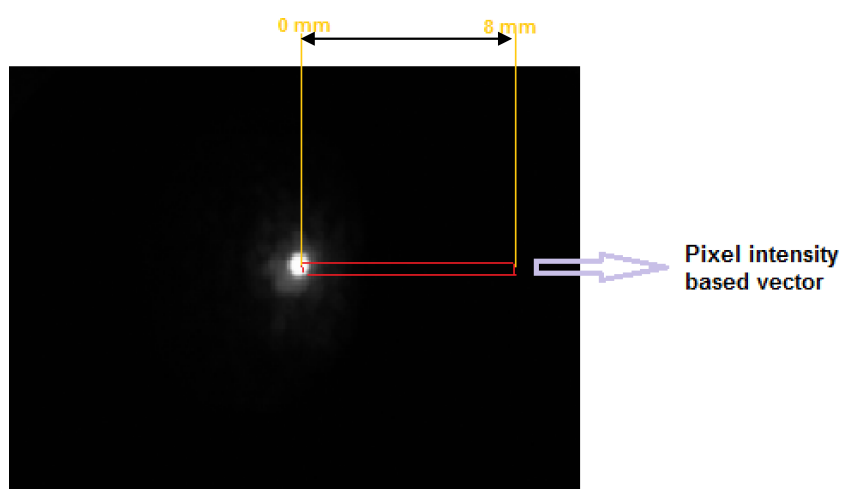

(a) have different optical properties are extracted from the transmission-based spectroscopic measurements in Fig. 3. Moreover, MC simulation results were dependent upon source-detector distance for each pair of $\mu_{a}$ and $\mu_{s}$. Thereby, while moving detector away from the source, it is seen that diffuse reflectance values exponentially decreased. In Fig. 5, we can easily see that the captured image of 6 th phantom regarding to Table 1 has a saturation region, even though an optical filter was used to attenuate high energy of laser light in our experimental setup. The measured absorption coefficient of 6 th phantom was $8,8373 \mathrm{~cm}^{-1}$ which closely equals to the absorption coefficient of human-skin tissue at $633 \mathrm{~nm} \cdot{ }^{20,21}$

Surface image of each phantom were handled and the results gave us that the extracted features from

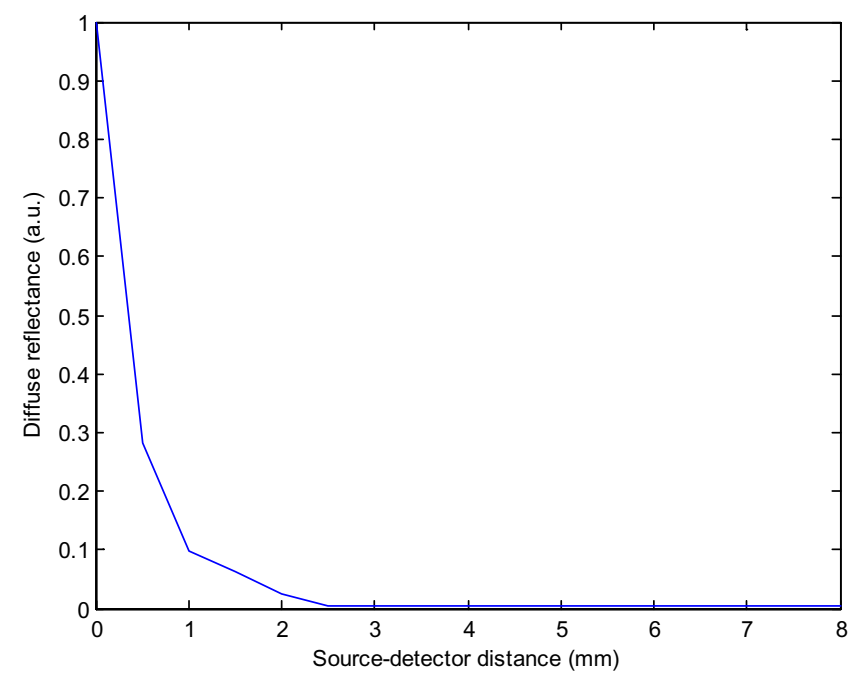

(b)

Fig. 5. The related image and corresponding reflectance spectrum for $\mu_{s}=1.4289 \mathrm{~cm}^{-1}, \mu_{a}=8.8373 \mathrm{~cm}^{-1}$. (a) 6 th phantom in Table 1 after applying image processing. (b) Spatially resolved diffuse reflectance versus distance for (a). 


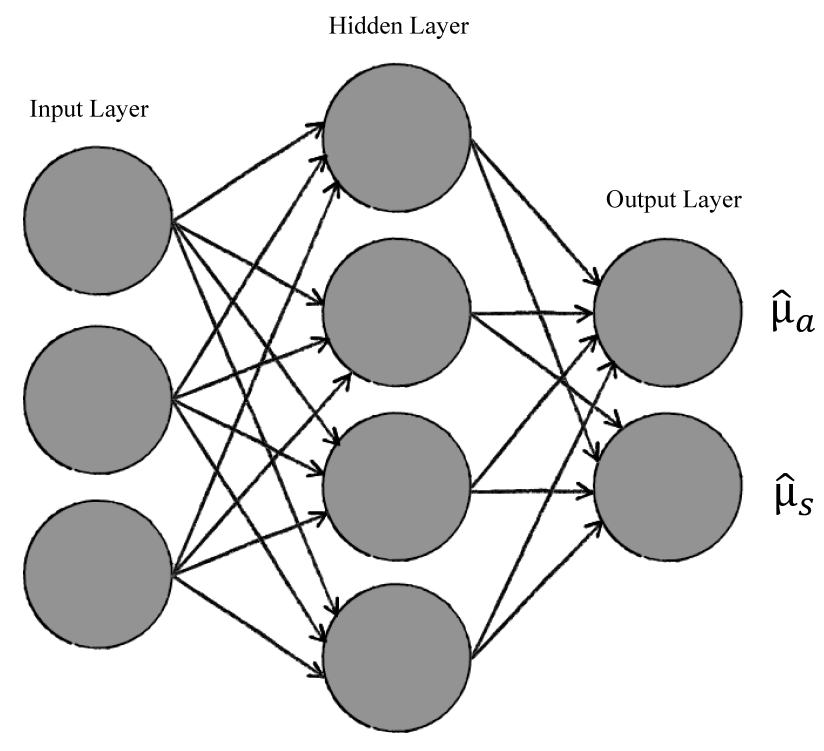

Fig. 6. ANN structure for the estimation of $\mu_{a}$ and $\mu_{s}$.

each image contain a normalized pixel intensities that can be considered as diffuse reflectance decreased exponentially for $0-8 \mathrm{~mm}$ distance. On the other hand, for inverse modeling of obtained spectra, we modeled an ANN structure as depicted in Fig. 6. We firstly considered the MC simulation results for $0-8 \mathrm{~mm}$ source-detector distance in terms of conducting neural network for training-testing process. Next, imagebased optical vectors (or spectra) were used for training-testing the network in the same manner. However, for the estimation of optical properties of the liquid phantoms, we took into account only the estimation of $\mu_{a}$ while conducting ANN as shown in Figs. 7 and 8. That is

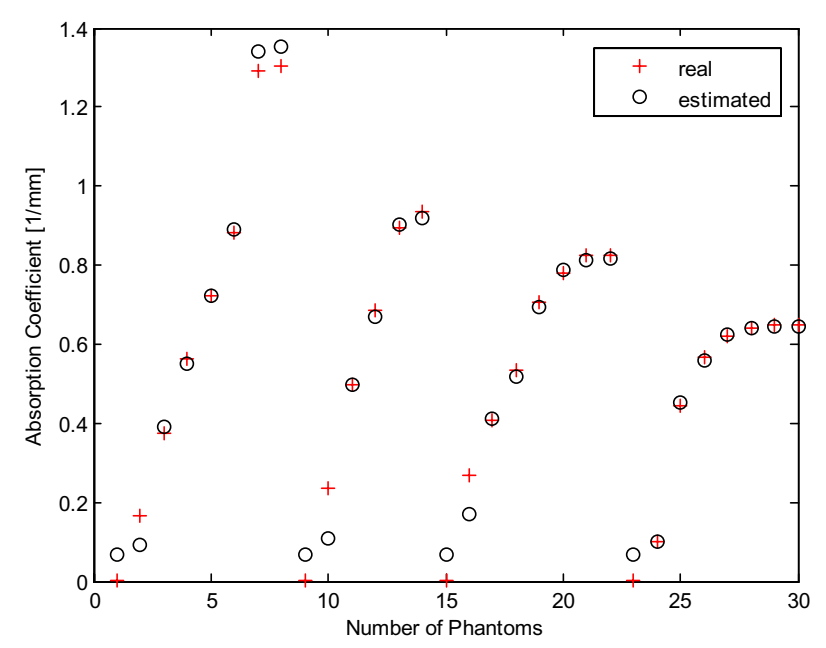

Fig. 7. Estimation of $\mu_{a}$ using MC simulation-based diffuse reflectance.

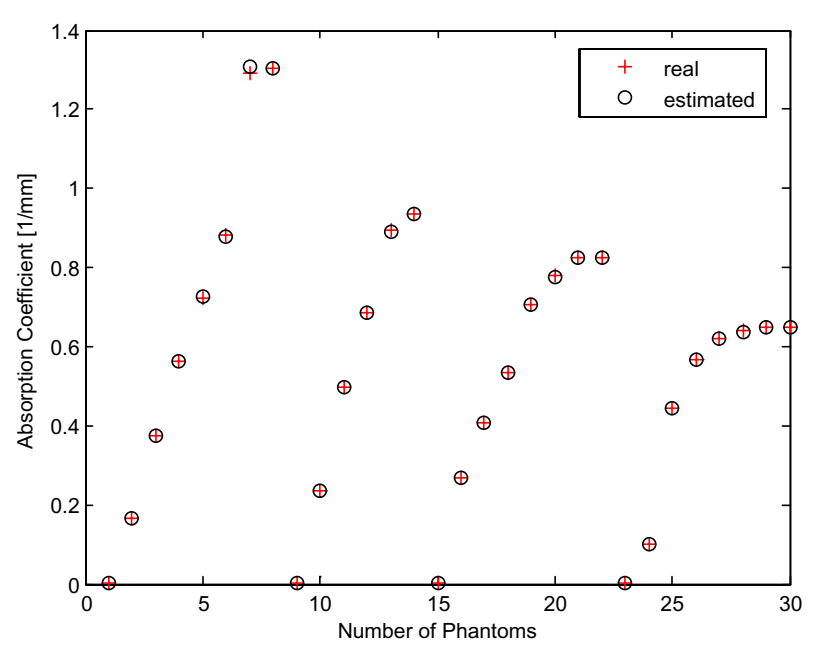

Fig. 8. Estimation of $\mu_{a}$ using image-based diffuse reflectance.

because, each phantom group has a fixed $\mu_{s}$ value, so the use of ANN structure for these $\mu_{s}$ values in Table 1 is not meaningful.

In Fig. 7, it is shown that all MC simulation outputs were used to estimate $\mu_{a}$ values by ANN. During learning mode, Levenberg-Marquardt algorithm was used such that $60 \%$ of diffuse reflectance values was for testing and $35 \%$ was for training and finally, we validated with $5 \%$ part of all values using cross-validation technique. Also, the number of hidden layers was 10 . At this point, the input variables are presented to the network and estimated output variables were compared with the desired output (real) variables. The output errors, which are the differences between the estimated and the desired values, are then used to adjust the connection weights in the network. Thereby, mean square error (MSE) was calculated as 0.0019 . In Fig. 8, image-based data that has 30 spectrum corresponding to the Table 1 were used for the estimation of $\mu_{a}$ and MSE was $1.24 \mathrm{e}-0.5$. In the same way, Levenberg-Marquardt algorithm was used in the learning mode and $70 \%$ of diffuse reflectance values was for testing and $25 \%$ was for training and finally, we validated with $5 \%$ part of all values. Also, the number of hidden layers was selected to 5 .

\section{Conclusions}

In this paper, we demonstrated ANNs based on the estimation of tissue optical properties by $5^{\circ}$ obliquecapturing diffuse reflectance. Our study was based on the two different approaches. The first one is MC simulation that relies on ensemble averaging of the 
data created depending on random sampling from a probability distribution. The next approach for obtaining experimental data is that we set up an optical assembly that contains laser light source at $633 \mathrm{~nm}$ wavelength, mirror, liquid phantoms and CMOS camera. By preparing samples, we measured absorbance quantities and then decomposed into scattering and absorption coefficients by setting up transmission spectroscopy. Latter, we input the calculated optical parameters for MC code. Using known optical properties of samples, we acquired images for 30 different phantoms, respectively according to Table 1. After applying image processing, 17 element-vectors that substitutes diffuse reflectance values were extracted from each single image. Finally, we modeled neural networks for inversion to separate diffuse reflectance into the absorption and scattering coefficients. MC simulation outcomes were assessed in itself through a network structure and image data were also evaluated in itself. At this point, it is seen that the neural network trained by MC data is more accurate than the neural network trained by image data for the determination of optical parameters from the diffuse reflectance. For our future studies, on behalf of MC modeling, the use of multitasking operating system with multicore Central Processing Unit ( $C P U$ ) may be an alternative way in terms of reducing code execution time ${ }^{12}$ instead of using GPU for acceleration of a source code. Also, in order to achieve a high performance MC simulation needs to be implemented in a parallel computing environment with multi/many core CPU units. For example, Ziegenhein et al. shows that CPU-based implementation on a modern workstation is between $1.25 \times$ and $1.95 \times$ faster than a well known GPU implementation of same simulation method on a NVIDIA Tesla C2050. ${ }^{22}$ On the other hand, providing a contribution to the ANN results due to training/testing, we will be planned to use the BrainOS ${ }^{17}$ approach with miniaturized robotics system to scan the surface of the human skin in order to model inner structure of tissue and finally to get optical parameters using machine learning algorithms in artificial intelligence.

\section{Acknowledgments}

This work was supported by the Scientific and Technological Research Council of Turkey (TUBITAK), under Grant No. 113E771.

\section{References}

1. L. V. Wang, H. Wu, Biomedical Optics Principles and Imaging, 1st edn. E-Publishing, New Jersey (2007).

2. L. Zhang, Z. Wang, M. Zhou, "Determination of the optical coefficients of biological tissue by neural network," J. Mod. Opt. 1163-1170 (2010).

3. L. Jacques, B. W. Pogue, "Tutorial on diffuse light transport,". J. Biomed. Opt. 041301-1-19 (2008).

4. L.Wang, L. Jacques, "Monte Carlo modeling of light transport in multi-layered tissues in standard C." University of Texas M. D. Anderson Cancer Center, Houston, Texas (1992).

5. P. Beard, "Biomedical photoacuostic imaging," Interface Focus 602-631 (2011).

6. Z. George, D. Aikaterini, "Modeling diffuse reflectance from homogeneous semi-infinite turbid media for biological tissue applications: A Monte Carlo study," Biomed. Opt. Express 3284-3292 (2011).

7. Z. Chaigang, L. Quan, "Review of Monte Carlo modeling of light transport in tissues," J. Biomed. Opt. doi: 10.1117/1.JBO.18.5.050902 (2013).

8. J. Mitra, Multi spectral image processing applied to dermatology, Master's. thesis (2009).

9. G. Zonios, A. Dimou, "Modeling diffuse reflectance from semi-infinite turbid media: Application to the study of skin optical properties," Opt. Express 86638664 (2006).

10. Y. Yong, P. Morgan, C. Pitter, "Characterization of layered scattering media using polarized light measurements and neural networks," J. Biomed. Opt. 504-506 (2003).

11. A. Cserkaszky, "Monte Carlo based PET image reconstruction on GPU," Master's thesis, Budapest, (2011).

12. B. Moyer, Real World Multiple Embedded Systems, 1st edn., SantaCruz, CA, USA (2013).

13. S. A. Prahl, M. Keijzer, S. L. Jacques, A. J. Welch, "A Monte Carlo model of light propagation in tissue," SPIE institute series 102-111 (1989).

14. L. Wang, S. L. Jacques, Zheng, $M C M L-M o n t e$ Carlo Modeling of Light Transport In Multi-Layered Tissues, pp. 131-146, Elsevier, Netherlands (1995).

15. S. E. Hernandez, V. D. Rodriquez, F. A. Martin, M. A. Castellano, J. S. Gonzalez, "Diffuse reflectance spectroscopy characterization of hemoglobin and intralipid solutions: in vitro measurements with continuous variation of absorption and scattering," J. Biomed. Opt. doi: 10.1117/1.3149864 (2009).

16. S. T. Flock, S. L. Jacques, B. C. Wilson, W. M. Star, M. J. C. Van Gemert, "Optical Properties of Intralipid: A phantom medium for light propagation studies," LasersSurg. Med. 510-518 (1992). 
17. E. M. Izhikevich, http://www.technologyreview. com/news/530871/robots-that-learn-through-repetition-not-programming/, MIT Technology Review (2014).

18. R. O. Duda, P. E. Hart, D. G. Stork, Pattern Classification, 2nd edn. California (1973).

19. S. A. Sullivan, "Experimental study of the absorption in distilled water, artificial seawater and heavy water in the visible region of the spectrum," Opt. Soc. Am. J. 962-968 (1963).

20. S. M. Jacques, "Optical properties of biological tissues: A review," Phys. Med. Biol. R37-R61 (2013).

21. A. N. Bashkatov, E. A. Genina, V. I. Kochubey, V. V. Tuchin, "Optical properties of human skin, subcutaneous and mucous tissues in the wavelength range from 400 to 2000 nm," J. Phys. D: Appl. Phys. 2543-2555 (2005).

22. P. Ziengenhein, S. Pirner, Ph. C. Kamerling, U. Oelfke, "Fast CPU-based Monte Carlo simulation for radio therapy dose calculation," Phys. Med. Biol. 6097-6111 (2015).

23. M. Riedmiller, H. Braun, "A Direct Adaptive Method for Faster Backpropagation Learning: The RPROP Algorithm," IEEE 586-591 (1993).

24. T. Lister, A. P. Wright, H. P. Chappell, "Optical properties of human skin," J. Bio. Opt. (2012). 